\title{
Recent Implementations of Fiber and Integrated Tunable Microwave Photonics Filters
}

\author{
Juan Sancho, Juan Lloret, N. Primerov*, Salvador Sales, L. Thévenaz* and José Capmany \\ ITEAM Research Institute, Optical and Quantum Communications Group, Universidad Politécnica de Valencia, \\ Camino de Vera s/n, 46022 Valencia, Spain, juasandu@iteam.upv.es \\ * Ecole Polytechnique Fédérale de Lausanne, Institute of Electrical Engineering, Lausanne, Switzerland
}

\begin{abstract}
We present recent implementations of microwave photonic filters using the following two technologies: InP-based MDR (microdisk resonator) on and coupled to a nanophotonic SOI waveguide and dynamic Brillouin gratings (DBGs) in polarization maintaining fibers (PMF). Quasi-linear and continuously tunable $\sim 360^{\circ}$ phase shifts have been experimentally obtained when considering radiofrequencies greater than $18 \mathrm{GHz}$ using MDR. Besides, we have designed and experimentally demonstrated multi-tap MWP filters, based on dynamic Brillouin grating reflectors in polarization maintaining fibers, showing a FSR variation larger than $60 \%$ in a two-tap filter.
\end{abstract}

Keywords: Analog optical signal processing; Integrated optics devices; Scattering, stimulated Brillouin; Nonlinear optics, fibers.

\section{INTRODUCTION}

Microwave photonic filters $[1,2]$ have attracted the interest from both the research community and the industrial sector over the last decades. Microwave photonic filters enable the realization of functionalities in microwave systems that are either complex or even not directly possible in the radiofrequency domain. Fast reconfigurability and tuneability, large bandwidth, immunity to electromagnetic interference (EMI), low losses, small size and weight are highly desirable features, which are however not straightforward to implement using traditional electronic circuitry.

Microwave photonic filters use to be based on a number of weighted and delayed samples of the RF signal are produced in the optical domain and combined upon detection. In particular, finite impulse response (FIR) [2] filters combine at their output a finite set of delayed and weighted replicas or taps of the input optical signal while infinite impulse response (IRR) are based on recirculating cavities to provide an infinite number of weighted and delayed replicas of the input optical signal [2]. Both implementations imply a periodic response in the frequency domain. The period, known as Free Spectral Range (FSR), is given by the inverse of the basic delay between consecutive samples, $T$. To achieve the desired tuneability and reconfigurability of the filter, it is necessary to find the mechanisms to vary $T$. Besides, the amplitude of the weighted replicas can be changed by inserting loss/gain and some microwave phase shift can be tuned, too. The focus of this paper is going to be on the first results reported with the following two technologies: InP-based MDR (microdisk resonator) on and coupled to a nanophotonic SOI waveguide and dynamic Brillouin gratings (DBGs) in polarization maintaining fibers (PMF).

Silicon Photonics has emerged as the most preferable technology platform for developing several integrated optics based functionalities in the last decades [1]. Silicon-based technology can benefit from economics of scale for mass-market applications, resulting in low-cost and CMOS-compatible solutions [5, 6]. However, a major obstacle for large-scale silicon-based electronic-photonic integration is the indirect band gap of silicon. A very promising solution for implementing active functionalities using SOI platforms consists of bonding on a direct band gap material, especially those belonging to the III-V group. We present the microwave photonic application of an InP-based MDR heterogeneously integrated on and coupled to a nanophotonic SOI waveguide. Firstly, a MWP fully-tunable phase shifter based on the cited technology is presented and demonstrated. Finally, the phase shifter is used as a key element in a notch-type filtering scheme yielding distortion-free and wideband response with a tuning range $\sim 100 \%$ over the free spectral range (FSR).

Meanwhile, a novel method to generate long variable optical delays of broadband signals has been proposed [10-11]. It makes use of stimulated Brillouin scattering (SBS) interaction between two pumps that are copolarized along one principal axis of a polarization maintaining fiber (PMF) [12-14]. The interaction generates a so-called dynamic Brillouin grating (DBG), which in turn could reflect incoming probe signals that are polarized along the orthogonal principal axis. DBGs are essentially optical wave reflectors that can be created at any preset position along the entire PMF. We demonstrate the realization of MWP filters using DBGs. A multitap performance is demonstrated, where the combined reflections from multiple DBGs along a PMF are employed. 


\section{MWP FILTER USING A SINGLE HETEROGENEOUSLY INTEGRATED III-V/SOI-MICRODISK}

To date, III-V/SOI microdisk resonators (MDR) have been used to develop several all-optical signal processing involving digital signals [3]. However, the potential of III-V/SOI based MDR enabling MWP functionalities was unexplored so far. We are going to show the suitability of exploiting MDR in the implementation of photonic RF phase shifters and MWP filters. The III-V/SOI MDR consists of an InP cavity which is integrated on and coupled to a SOI waveguide. The principle of operation is based on using OSSB modulation combined by a single over-coupled MDR. By acting on the carrier density in the InP cavity, the effective index can be modified. As a consequence, the spectral placement of the notches can be shifted. In this manner, the phase induced on the optical carrier can be continuously adjusted, which at the end results in the phase control of the RF signal. This concept is shown in Fig. 1.

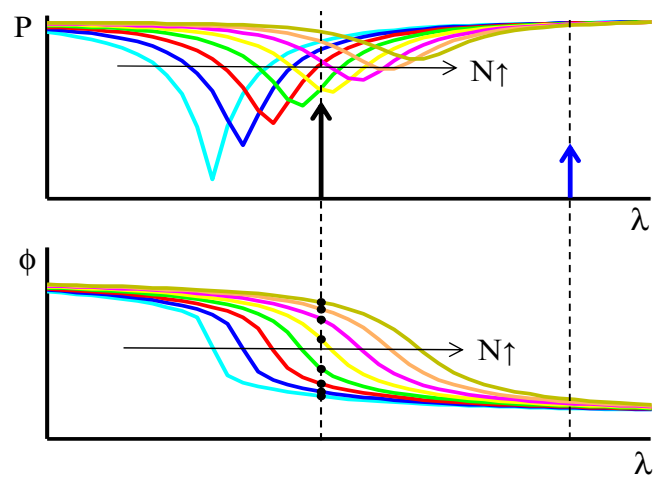

Figure 1. Principle of operation as a photonic RF phase shifter.

Figure 2 shows the spectral placement for both the optical carrier and the modulation sideband within the amplitude and phase transfer functions in the vicinity of a resonance as a function of the injection current into the MDR. The functionality as a photonic RF phase shifter in terms of phase shift and power variation for different RF frequencies is depicted in Fig. 2(b). A minimum frequency of $18 \mathrm{GHz}$ is needed to reach quasi-linear and continuously tunable $360^{\circ}$. The tunability speed is limited by the carrier dynamics in the InP, which results in a great enhancement with respect to other solutions [4-5]. Moreover, to the best of our knowledge, is the smallest MWP phase shifter reported up to now.
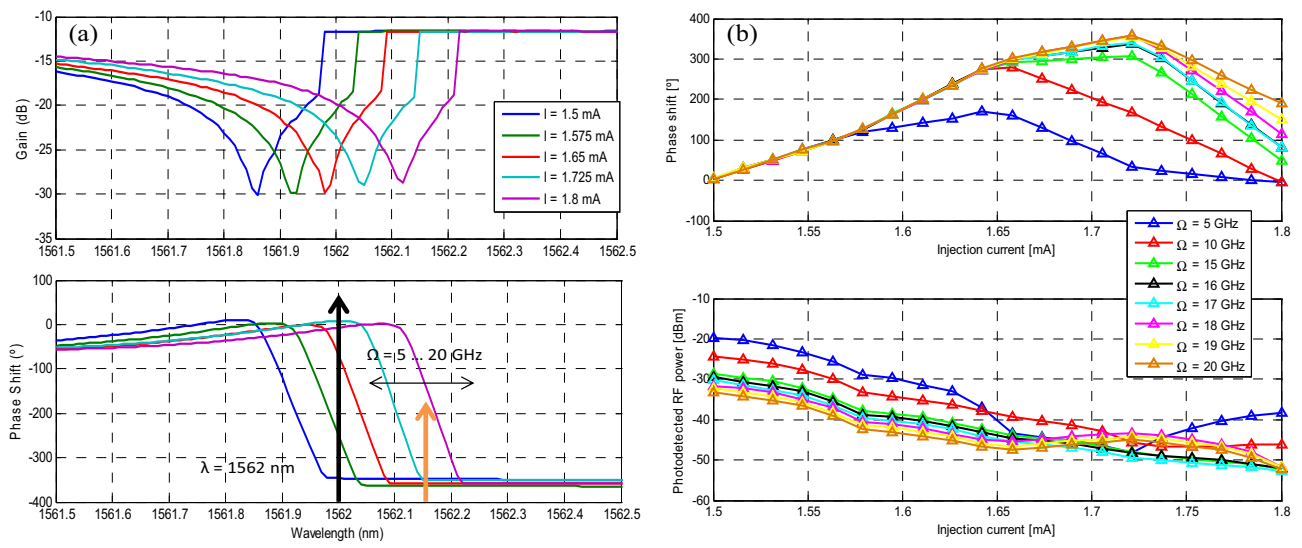

Figure 2: (a) Amplitude and phase transfer functions of the MDR;

(b) Phase shift and power variation of the RF signal.

The photonic RF phase shifter functionality was exploited in the implementation of tunable complex-valued MWP filters. A proof-of-concept notch-type filter was implemented at the central operating frequency of $20 \mathrm{GHz}$. The MDR is inserted in the lower arm of the interferometric structure. In this manner, the basic phase shift between both taps can be controlled by properly adjusting the injection current into the MDR. Figure 3(a) shows the experimental setup. The interferometric structure is characterized by a length imbalance of $1.27 \mathrm{~m}$, which corresponds to a notch-type response with a FSR of roughly $163 \mathrm{MHz}$. At the output of the IM, the OSSB signal is amplified by means of an erbium doped fiber amplifier (EDFA). An optical 0.8-nm-wide bandpass filter (OBPF) centered at the carrier wavelength is also used for noise suppression purposes. To avoid coherent-related problems, a balanced photodetector (BPD) is used. In order to obtain the maximum tunability range, the center frequency of the filter must be high enough to be phase-shifted up to $360^{\circ}$. Figure 3(b) displays the normalized filter frequency response for different injection currents into the MDR. Experimental (symbols) and theoretical (solid lines) results show a good agreement. The small deviations are attributed to reflections in the electrical 
part of the setup, the residual unwanted sideband in the OSSB modulation and the spontaneous noise. Nearly $2 \pi$ controllable basic phase shift $(\Delta \square)$ over the operating bandwidth leads to continuously $\sim 100 \%$ fractional tuning of the filter response. Distortion-free response and fractional tuning of $100 \%$ was demonstrated.
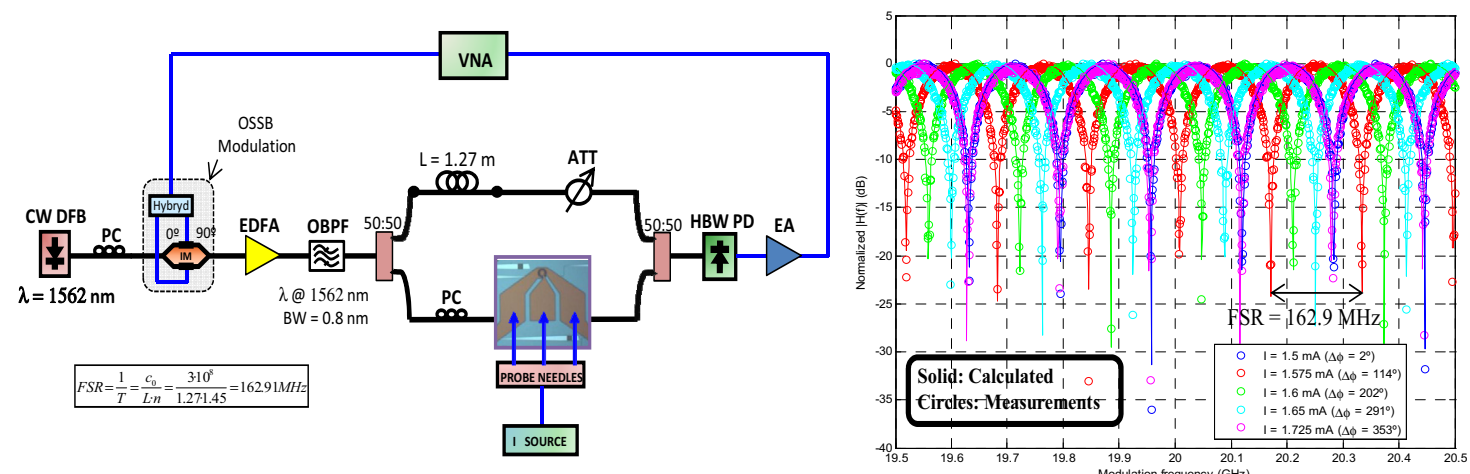

Figure 3: (a) Amplitude and phase transfer functions of the MDR;

(b) Phase shift and power variation of the RF signal.

\section{TUNABLE MWP FILTER BASED ON DYNAMIC BRILLOUIN GRATINGS}

MWP filters based on discrete time signal processing principles comprise several optical delay lines which provide and combine at the output of the system a set of delayed and weighted replicas of the input signal. One novel technique to achieve reconfigurable signal delays over a wide range has been demonstrated in [6]. It makes use of dynamic Brillouin gratings (DBGs) in polarization maintaining fibers (PMF), where the gratings acting as reflectors can be created at any present position along the fiber. Combined reflections from different dynamic gratings are used for implementing multi-tap filters with a variable free spectral range (FSR). Different DBGbased filter configurations have been developed through join collaboration between UPVLC and EPFL.

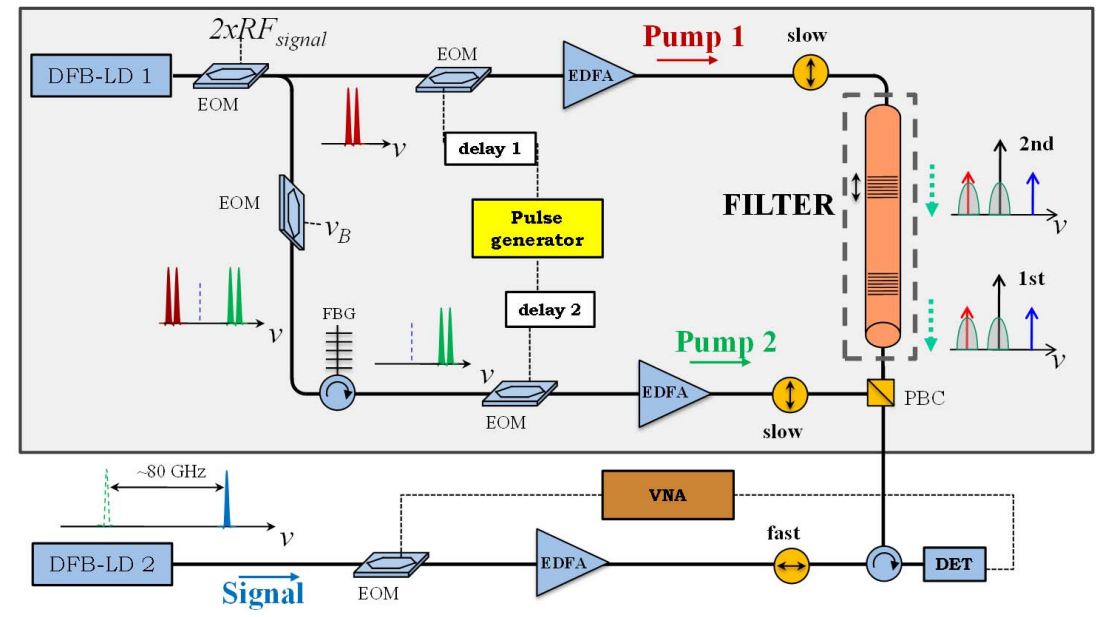

Figure 4. Experimental setup.

Figure 4 illustrates the experimental layout of the DBG-based MWP filter. The DBG generation appears only at positions along the PMF fiber where two pump pulses are simultaneously present. Both pumps have to be polarized along the slow axis. The present configuration allows the generation of two gratings within the same physical position but also different pith. Carrier and left sideband are reflected for different grating which means no RF central frequency limitations. A microwave signal acting as a probe has been linearly polarized along the fast axis and spectrally detuned $(\sim 80 \mathrm{GHz})$ due to the birefringence of the PMF fiber. Since the entire probe reflections are phase conjugated [7], it is expected that the delayed replicas of the microwave signal emerge out of the fiber with equal phases. The frequency response of the reflections combined is therefore equivalent to that of a multi-tap filter with equal, positive weights. The number of taps comprising the filter is readily reconfigurable by simply changing the number of dynamic grating reflectors positioned in the fiber.

Different filter frequency responses attending two, three and four-tap implementations within $\sim 110 \mathrm{~m} \mathrm{PMF}$ fiber are represented in Fig. 5. Around 65\% of FSR tunability within $1 \mathrm{GHz}$ bandwidth is achieved for the twotap approach. The tunability is carried out through sweeping the repetition rate of the two pulse trains used to build up the gratings. The four-tap case doesn't permit any FSR variation since the fiber length limit is reached. The experimental and theoretical misalignment is mainly due to the non-uniform Brillouin frequency and birefringence along the PMF fiber. 


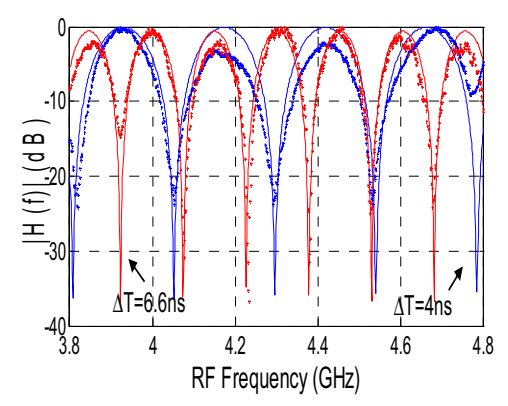

(a)

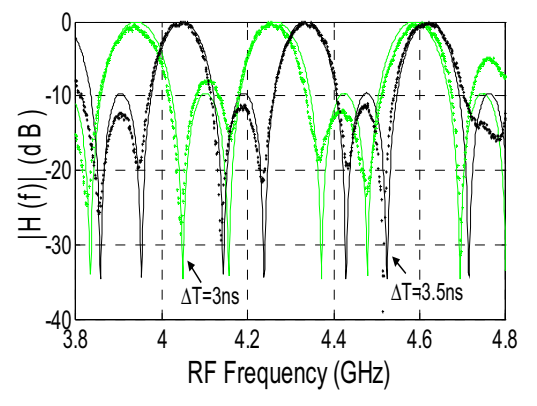

(b)

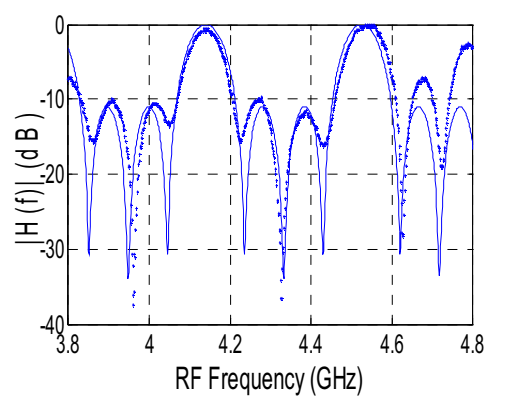

(c)

Figure 5. Frequency response of the: (a) two-tap, (b) three-tap and (c) four-tap filter configuration.

\section{CONCLUSIONS}

An ultra-small and low-power broadband MWP phase shifter based on a single III-V/SOI MDR in combination with OSSB modulation has been proposed and demonstrated. Quasi-linear and continuously tunable $\sim 360^{\circ}$ phase shifts have been experimentally obtained when considering radiofrequencies greater than $18 \mathrm{GHz}$. This phase shifter has been used for implementing complex-valued coefficients in tunable MWP filtering schemes. A proofof-concept implementation involving two taps is demonstrated. Distortion-free and high-bandwidth filter responses with tuning range of $\sim 100 \%$ over the FSR have been obtained.

Besides, we have designed and experimentally demonstrated multi-tap MWP filters, based on dynamic Brillouin grating reflectors in polarization maintaining fibers. The spectral responses of the proposed novel variety of filters can be dynamically tuned and reconfigured, by simply changing the characteristics of single or multiple DBG generations, showing a FSR variation larger than $60 \%$ in a two-tap filter.

\section{ACKNOWLEDGEMENTS}

The authors wish to acknowledge the financial support of the European Community's Seventh Framework Programme (FP 7) project GOSPEL; the GVA PROMETEO 2008/092, Infraestructura FEDER UPVOV08-3E008, the Plan Nacional I + D TEC2011-29120-C05-05.

\section{REFERENCES}

[1] J. Capmany and D. Novak, "Microwave photonics combines two worlds," Nature Photon. 1, 319-330 (2007).

[2] J. Capmany, B. Ortega, D. Pastor and S. Sales, "Discrete-time optical processing of microwave signals," IEEE J. Lightwave Technol. 23, 702-723 (2005).

[3] D. Van Thourhout, et al., "Nanophotonic devices for optical interconnect," J. Select. Top. Quantum Electron. 16(5), 1363-1375 (2010).

[4] M. Pu, et al., "Tunable microwave phase shifter based on silicon-on-insulator microring resonator," Photon. Technol. Lett. 22(12), 869-871 (2010).

[5] M. Pu, et al., "Widely tunable microwave phase shifter based on silicon-on-insulator dual-microring resonator," Opt. Exp. 18(6), 6172-6182 (2010).

[6] N. Primerov, et al., "Ultra wide range tunable delay line using dynamic grating reflectors in optical fibers," in Proc. OFC'10, San Diego, USA (2010).

[7] K. Y. Song, et al., "All-optical dynamic grating generation based on Brillouin scattering in polarization maintaining fiber," Opt. Lett. 33(9), 926-928 (2008). 